\title{
Broadband Nonlinear Optical Response of Monolayer MoSe under Ultrafast Excitation
}

\author{
Zhonghui $\mathrm{Nie}^{1}$, Chiara Trovatello², Eva A.A. Pogna 2 , Stefano Dal Conte ${ }^{2},{ }^{3}$, Paulo B. \\ Miranda $^{6}$, Edmund Kelleher ${ }^{4}$, Chunhui Zhu ${ }^{1}$, Edmond Turcu ${ }^{1}$, Yongbing $\mathrm{Xu}^{1}$, Kaihui \\ Liu $^{5}$, Giulio Cerullo ${ }^{2,3^{*}}$ and Fengqiu Wang ${ }^{*}$
}

${ }^{1}$ School of Electronic Science and Engineering and Collaborative Innovation Center of Advanced Microstructures, Nanjing University, 210093, China

${ }^{2}$ Dipartimento di Fisica, Politecnico di Milano, Piazza Leonardo da Vinci 32, I-20133 Milano, Italy

${ }^{3}$ Istituto di Fotonica e Nanotecnologie (IFN), CNR, Piazza Leonardo da Vinci 32, I-20133

Milano, Italy

${ }^{4}$ Femtosecond Optics Group, Department of Physics, Imperial College London, SW7 2AZ, UK

${ }^{5}$ State Key Laboratory for Mesoscopic Physics, School of Physics, Peking University, Beijing 100871,

China

${ }^{6}$ Instituto de Física de São Carlos, Universidade de São Paulo, P.O. Box 369, 13560-970 São Carlos-

SP, Brazil

Emails: fwang@nju.edu.cn, giulio.cerullo@fisi.polimi.it 


\begin{abstract}
:
Due to their strong light-matter interaction, monolayer transition metal dichalcogenides (TMDs) have proven to be promising candidates for nonlinear optics and optoelectronics. Here, we characterize the nonlinear absorption of CVD-grown monolayer $\mathrm{MoSe}_{2}$ in the $720-810 \mathrm{~nm}$ wavelength range. Surprisingly, despite the presence of strong exciton resonances, monolayer $\mathrm{MoSe}_{2}$ exhibits a uniform modulation depth of $\sim 80 \pm 3 \%$ and saturation intensity of $\sim 2.5 \pm 0.4 \mathrm{MW} / \mathrm{cm}^{2}$. In addition, pump-probe spectroscopy is performed to confirm the saturable absorption and reveal the photocarrier relaxation dynamics over hundreds of picoseconds. Our results for the first time unravel the unique broadband nonlinear absorptive behavior of monolayer $\mathrm{MoSe}_{2}$ under ultrafast excitation and highlight the potential of using monolayer TMDs as broadband ultrafast optical switches with customizable saturable absorption characteristics.
\end{abstract}


Atomically thin transition metal dichalcogenides (TMDs) have received significant attention because of their unique low-dimensional physics $^{1-6}$. Contrary to graphene, monolayer TMDs are semiconductors exhibiting a direct bandgap in the visible to near-infrared (NIR) range. The low dimensionality, strong quantum confinement and greatly enhanced Coulomb interactions ${ }^{3-4}$ are responsible for many peculiar properties of TMDs, such as their giant nonlinear optical response ${ }^{6}$. TMDs have been exploited to realize practical optoelectronic devices ${ }^{7-9}$, such as $\operatorname{LEDs}^{8}$, photodetectors $^{9}$, and ultrafast optical switches ${ }^{10}$. In particular, saturable absorbers (SAs) based on TMDs have been widely used to generate ultrashort pulses by either Q-switching or mode-locking ${ }^{11-14}$. For instance, Woodward et al. reported that a free-standing multilayer $\mathrm{MoS}_{2} \mathrm{SA}$, fabricated by liquid-phase exfoliation (LPE), can be adopted to Q-switch fiber lasers at $1060 \mathrm{~nm}, 1550 \mathrm{~nm}$ and $1924 \mathrm{~nm}^{13}$. TMD-based SAs usually employ liquid-processed nano-flakes in dispersions or in a polymeric matrix ${ }^{11-14}$. The nonlinear optical response of these samples has been found to be dependent on flake size and thickness ${ }^{15}$, and is strongly affected by extrinsic crystal properties, such as layer inhomogeneities, impurities, and defects ${ }^{16}$. These extrinsic factors lead to the existence of sub-bandgap, defect or traps states, therefore making it difficult to achieve full control of the saturable absorption behavior of TMD-based SAs.

By contrast, chemical vapour deposition (CVD) grown monolayer TMDs exhibit synthesis scalability, better layer control and much reduced defect densities. They represent a more desirable form for practical exploitation and have been successfully applied in mode-locked lasers ${ }^{17}$. On the other hand, the strong visible light absorption of monolayer TMDs, strongly renormalized by excitonic effects, makes their saturable absorption behavior in this wavelength region potentially more stable and 
controllable. Recently, few-layer TMDs have been reported as SAs for Q-switched visible lasers ${ }^{18}$. To achieve mode-locked operation in the visible range, it is desirable to finely control the nonlinear optical response of the SAs. The intrinsic nonlinear absorption of monolayer TMDs around the excitonic resonances in the visible-NIR range, including the key parameters of SAs, i.e. modulation depth, saturation intensity and recovery time, have yet to be experimentally characterized.

Here, we combine microscope-based nonlinear absorption and ultrafast degenerate pump-probe spectroscopy to study the nonlinear optical response of monolayer $\mathrm{MoSe}_{2}$ in a broad wavelength region, covering the exciton peaks. Nonlinear absorption measurements reveal a large modulation depth and a desirably small saturation intensity. Interestingly, both figures of merit remain constant over the entire probed wavelength range. This behavior is remarkably different from that of other exciton-dominated low-dimensional systems, such as carbon nanotubes ${ }^{19}$, and the uniform response allows to precisely deduce the nonlinear absorption saturation from a simple linear absorption measurement. Ultrafast degenerate pump-probe spectroscopy is further performed to confirm saturation of absorption and to illustrate the overall transient behavior of monolayer $\mathrm{MoSe}_{2}$.

The monolayer $\mathrm{MoSe}_{2}$ sample is grown on a $\mathrm{SiO}_{2} / \mathrm{Si}$ substrate by CVD, and it is then transferred onto a silver mirror by a wet-transfer technique based on a sacrificial layer of poly-methyl-methacrylate (PMMA). It should be noted that in our investigation we transfer the samples to a silver mirror to form a reflective geometry, just like the semiconductor saturable absorption mirror (SESAM). This would also allow us to directly probe the absorption feature of the $\mathrm{MoSe}_{2}$ sample. The protective layer on the silver mirror is about $100 \mathrm{~nm}$ thick, therefore the standing wave effect can be largely neglected. Fig. 
1(b) shows an optical micrograph, highlighting the triangular islands of monolayer $\mathrm{MoSe}_{2}$. The average lateral dimension of these monolayers is approximately $30-40 \mu \mathrm{m}$, larger than what is typically achieved using mechanical exfoliation, and atomic force spectroscopy (AFM) also indicates that the thickness of sample is about $0.75 \mathrm{~nm}$, consistent with previous reports. Fig. 1(c) and Fig. 1(d) show the linear absorption, the photoluminescence (PL) and the Raman spectra of the sample, respectively. The linear absorption spectrum shows two peaks centered at 790 and $700 \mathrm{~nm}$, corresponding to the $\mathrm{A}$ and $\mathrm{B}$ excitons, respectively. The PL peak of the samples is located at $\sim 790 \mathrm{~nm}$, and it corresponds to the radiative recombination of the lowest-energy exciton. The two distinct peaks observed in the Raman spectrum (Fig. 1d) correspond to the out-of-plane $A_{1 g}$ and in-plane $E_{2 g}$ vibrational mode. In our experiment, $\mathrm{A}_{1 \mathrm{~g}}$ and $\mathrm{E}_{2 \mathrm{~g}}$ peaks are at $240.9 \mathrm{~cm}^{-1}$ and $287.8 \mathrm{~cm}^{-1}$, consistent with results previously obtained ${ }^{20}$.

We first recorded the nonlinear optical response of monolayer $\mathrm{MoSe}_{2}$, while tuning the pump wavelength through its excitonic resonance. Fig. 2(a) shows the intensity dependent nonlinear reflectance of the sample measured by a single-pulse spectroscopy setup, shown in Fig. 1(a), coupled with a microscope, where a tunable Ti:Sapphire laser $(80-\mathrm{MHz}$ repetition rate, $150-\mathrm{fs}$ pulse width) is focused on the sample by a 40X objective over a spot size of $2 \mu \mathrm{m}$ and the portion of beam reflected by the sample is recorded by a photodiode. We found that monolayer $\mathrm{MoSe}_{2}$ exhibits saturable absorption within the measured range. The absorption strongly depends on the intensity and as the excitation wavelength moves towards the exciton resonance, an increase in the change of reflectance is 
obtained. While the largest change in reflectance can reach $25 \%$ for on-resonance pumping $(790 \mathrm{~nm}$ ), this can be as low as 5\% for the off-resonance wavelengths, for instance at $812 \mathrm{~nm}$.

Through the Lambert-Beer's law, the absorbance of monolayer $\mathrm{MoSe}_{2}$, as shown in the inset of Fig. 2(b), can be calculated from the reflectance data. Then, the nonlinear absorption data as a function of the pumping intensity $I$, is fitted using a simple two-level saturable absorption model that has been widely used $^{21}$ :

$$
\alpha(I)=\frac{\alpha_{0}}{1+I / I_{s a t}}+\alpha_{n s}
$$

where $\alpha(I)$ is the absorption coefficient, $\alpha_{0}$ and $\alpha_{n s}$ represent saturable and nonsaturable absorption components respectively. $\Delta=\frac{\alpha_{0}}{\alpha_{0}+\alpha_{n s}}$ is the modulation depth and $I_{s a t}$ is the saturation intensity, defined as the value of $I$ for which the saturable part of the absorption falls by one-half. It is found that, for all the wavelengths investigated, the modulation depth and the saturation intensity fall within the range of $\sim 80 \pm 3 \%$ and $\sim 2.5 \pm 0.4 \mathrm{MW} / \mathrm{cm}^{2}$, as shown in Fig. 2(b). The extracted parameters and their wavelength-independence demonstrate that monolayer $\mathrm{MoSe}_{2}$ could act as an excellent $\mathrm{SA}$ in the measured spectral range. Compared with some TMD SAs based on LPE ${ }^{12,14}$, the saturation intensity of monolayer $\mathrm{MoSe}_{2}$ is about two orders of magnitude lower while the modulation depth is appreciably higher ${ }^{11-15,18}$. The relatively large modulation depth observed here may be caused by the much smaller non-saturable loss of monolayer $\mathrm{MoSe}_{2}$, compared to SAs based on LPE samples. This advantage has also been observed in monolayer graphene ${ }^{21,22}$, which shows a high modulation depth of $\sim 66.5 \%$ and a saturation intensity $\sim 0.71 \mathrm{MW} / \mathrm{cm}^{2}$ in the near-infrared frequency range $\mathrm{e}^{21}$. 
Interestingly, the normalized absorption curves at different excitation photon energies almost completely overlap over the broad probed wavelength range, as it is shown in Fig. 2(b). This behavior is significantly different from that expected for systems characterized by high-binding energy excitons, e.g. single-walled carbon nanotubes, where the modulation depth, by contrast, exhibits a monotonic increase approaching the excitonic resonance ${ }^{19}$. The observation of constant nonlinear absorption parameters across a broad optical spectrum has an important implication for the saturable absorber design: it allows to deduce the nonlinear absorption behavior at an arbitrary wavelength simply from the linear absorbance value, i.e. using Equation (1). The observed uniformity of the nonlinear absorption can be traced back to the excitation wavelength independence of the non-equilibrium optical response measured in monolayer TMDs ${ }^{23}$. As the pulse duration of laser used is around $150 \mathrm{fs}$ and it has been recently found that excitons formation process occurs on the same or shorter time scale ${ }^{24}$, our uniform absorption therefore suggests highly similar thermalization dynamics over the wavelength investigated.

In addition to modulation depth and saturation intensity, a fundamental property defining the performances of a semiconductor SA is the recovery time. In particular, the dynamics of the onset of the mode-locking regime and the duration of the generated pulse are strongly dependent on the relaxation dynamics of the $\mathrm{SA}^{25}$. In order to characterize it, we monitor the relaxation to equilibrium of the monolayer $\mathrm{MoSe}_{2}$ flakes by means of degenerate pump-probe spectroscopy. Tunable pump and probe pulses, with $\approx 10$-nm bandwidth and $\approx 100$-fs duration, are produced by an optical parametric amplifier pumped by a regeneratively amplified Ti:sapphire laser at $2-\mathrm{kHz}$ repetition rate. 
Fig. 3(a) shows the recorded normalized differential reflection signal $\Delta R / R(t)$ for a representative wavelength $(760 \mathrm{~nm}$, at the center of the investigated tuning range) as a function of the pump-probe time delay, for different incident pump intensities. The relaxation dynamics are best fitted with a tripleexponential decay function, with time constants $\left(\tau_{1}=621 \pm 13 \mathrm{fs}, \tau_{2}=18 \pm 1 \mathrm{ps}\right.$ and $\left.\tau_{3}=83 \pm 8 \mathrm{ps}\right)-$ slightly dependent on the excitation fluence - such that the system recovery is completed in less than $300 \mathrm{ps}^{26}$. The differential reflection signal rises instantaneously within the $100 \mathrm{fs}$ time resolution of the measurement, as shown in the inset of Fig. 3(a)). The transient increase of reflection after photoexcitation has been attributed to both the bleaching of the exciton resonance due to Pauli blocking mechanism and to photo-induced bandgap renormalization effects ${ }^{23}$. In this high excitation regime, still well below Mott's transition threshold for TMDs ${ }^{27}$, several mechanisms for the decay of the photoinduced exciton population are active: excitons dissociation into free carriers ${ }^{28}$, interband recombination and exciton-exciton annihilation ${ }^{29,30}$. The ultrafast decay process shows a longer relaxation time, than that of LPE-based SAs, which can be an advantage in supporting self-starting operation of the modelocking regime ${ }^{25}$.

In addition to the extracted characteristic recovery time, pump-probe results confirm the strong saturable absorption of monolayer $\mathrm{MoSe}_{2}$, as indicated by the observed sublinear trend of the signal with the pump intensity shown in Fig. 3(b). Upon increasing the pump intensity, the differential reflectance saturates and the curve also suggests a large modulation depth, similar to the measured value of the nonlinear absorption. It should be noted that for pump-probe spectroscopy, a relatively high saturation intensity of few $\mathrm{GW} / \mathrm{cm}^{2}$ is deduced from Fig. 3(b). Such deviation of saturation intensities 
has been found in TMDs as well as other materials such as graphene ${ }^{21,22,32-34}$. Ref 31 provides a theoretical background for interpreting such a parameter consistency in SA characterization ${ }^{31}$. In the actual pump-probe measurement, the mismatch of the temporal and spatial overlap between pump and probe beams will result in higher intensity to reach saturation. In addition, difference of the the repetition rates of the laser used for the characterization (the $2 \mathrm{kHz}$ for pump-probe and $76 \mathrm{MHz}$ for the nonlinear absorption) may also have contributed to the difference in saturation intensity ${ }^{35}$.

In summary, ultrafast nonlinear absorption of monolayer $\mathrm{MoSe}_{2}$ under $\sim 150$ fs pulse excitation reveals a highly uniform response where the same modulation depth $(80 \pm 3 \%)$ and saturation intensity $\left(2.5 \pm 0.4 \mathrm{MW} / \mathrm{cm}^{2}\right)$ are found across the entire investigated frequency range, and pump-probe spectroscopy shows the hundred picoseconds recovery time of monolayer $\mathrm{MoSe}_{2}$. Our results provide useful guidelines for the design of novel photonic devices, in particular, ultrafast lasers working in the visible range. We stress that these findings may be straightforwardly generalized to other semiconducting TMDs.

Acknowledgements: This work was supported in part by the National Key R\&D Program of China (2017YFA0206304); the National Basic Research Program of China (2014CB921101 and 2011CB301900); National Natural Science Foundation of China (61775093, 61378025 and 61427812); National Young 1000 Talent Plan; A "Jiangsu Shuangchuang Team" Program; Jiangsu NSF (BK20170012 and BK20140054); and Australian Research Council (DP 160101474). GC acknowledges support by the European Union Horizon 2020 Programme under Grant Agreement No. 696656 Graphene Flagship. We thank Ruwen Peng for providing the linear absorption measurement. 


\section{References}

${ }^{1}$ K. F. Mark, C. Lee, J. Hone, and T. F. Heinz, Phys. Rev. Lett. 105, 136805 (2010).

${ }^{2}$ Y. Zhang, T. R. Chang, B. Zhou, Y. T. Cui, H. Han, Z. K. Liu, F. Schmitt, J. Lee, R. Moore, Y. L. Chen,

H. Lin, H. T. Jeng, S. K. Mo, Z. Hussain, A. Bansil, and Z. X. Sun, Nat. Nanotechnol. 9, 111-115 (2014).

${ }^{3}$ A. Chernikov, C. Ruppert, H. M. Hill, A. F. Rigosi, and T. F. Heinz, Nat. Photon. 9, 466-470 (2015).

${ }^{4}$ M. M. Ugeda, A. J. Bradley, S. F. Shi, F. H. da Jornada, Y. Zhang, D. Y. Qiu, W. Ruan, S. K. Mo, Z.

Hussain, Z. X. Shen, F. Wang, S. G. Louie, and M. F. Crommie, Nat. Mater. 13, 1091-1095 (2014).

${ }^{5}$ D. Xiao, G. B. Liu, F. Wang, X. D. Xu, and W. Yao, Phys. Rev. Lett. 108, 196802 (2012).

${ }^{6}$ X. B. Yin, Z. L. Ye, D. A. Chenet, Y. Ye, K. O’Brien, J. C. Hone, and X. Zhang, Science 344, 488-490 (2014).

${ }^{7}$ B. Radisavljevic, A. Radenovic, J. Brivio, V. Giacomentti, and A. Kis, Nat. Nanotechnol. 6, 147-150 (2011).

${ }^{8}$ J. S. Ross, P. Klement, A. M. Jones, N. J. Ghimire, J. Q. Yan, D. G. Mandrus, T. Taniguchi, K. Watanabe, K. Kitamura, W. Yao, D. H. Cobden, and X. D. Xu, Nat. Nanotechnol. 9, 268-272 (2014).

${ }^{9}$ O. Lopez-Sanchez, D. Lembke, M. Kayci, A. Radenovic, and A. Kis, Nat. Nanotechnol. 8, 497-501 (2013).

${ }^{10}$ Q. H. Wang, K. Kalantar-Zadeh, A. Kis, J. N. Coleman, and M. S. Strano, Nat. Nanotechnol. 7, 699-712, (2012).

${ }^{11}$ Z. Luo, Y. Li, M. Zhong. Y. Huang, X. Wan, J. Peng, and J. Weng, Photon. Res. 4, 231765 (2015)

${ }^{12}$ B. Chen, X. Zhang, K. Wu, H. Wang, J. Wang, and J. Chen, Opt. Express 23, 26723-26737 (2015). 
${ }^{13}$ R. I. Woodward, R. C. T. Howe, T. H. Runcorn, G. Hu, F. Torrisi, E. J. R. Kelleher, and T. Hasan, Opt. Express 23, 20051-20061 (2015).

${ }^{14}$ D. Mao, Y. Wang, C. Ma, L. Han, B. Jiang, X. Gan, S. Hua, W. Zhang, T. Mei and J. Zhao, Sci. Rep. 5 (2015).

${ }^{15}$ K. G. Zhou, M. Zhao, M. J. Chang, Q. Wang, X. Z. Wu, Y. Song, and H. L. Zhang, Small 11, 694$701(2015)$.

${ }^{16}$ M. Trushin, E. J. R. Kelleher, and T. Hasan, Phys. Rev. B 94, 155301 (2016).

${ }^{17}$ H. Xia, H. Li, C. Lan, C. Li, X. Zhang, S. Zhang, and Y. Liu, Opt. Express 22, 17341-17348 (2014).

${ }^{18}$ Z. Luo, D. Wu, B. Xu, H. Xu, Z. Cai, J. Peng, S. Xu, C. Zhu, F. Wang, Z. Sun, and H. Zhang, Nanoscale 8, 1066-1072 (2016).

${ }^{19}$ S. Xu, F. Wang, C. Zhu, Y. Meng, Y. Liu, W. Liu, J. Tang, K. Liu, G. Hu, R. C. T. Howe, T. Hasan, R. Zhang, Y. Shi, and Y. Xu, Nanoscale 8, 9304-9309 (2016).

${ }^{20}$ H. V. Han, A. Y. Lu, L. S. Lu, J. K. Huang, H. Li, C. L. Hsu, Y. C. Lin, M. H. Chiu, K. Suenaga, C. W. Chu, H. C. Kuo, W. H. Chang, L. J. Li, and Y. Shi, ACS Nano 10, 1454-1461 (2016).

${ }^{21}$ Q. Bao, H. Zhang, Y. Wang, Z. Ni, Y. Yan, Z. X. Shen, K. P. Loh, and D. Y. Tang, Adv. Funct. Mater. 19, 3077-3083 (2009).

${ }^{22}$ G. Xing, H. Guo, X. H. Zhang, T. C. Sum, and C. H. A. Huan, Opt. Express 18, 4564-4573 (2010).

${ }^{23}$ E. A. A. Pogna, M. Marsili, D. D. Fazio, S. D. Conte, C. Manzoni, D. Sangalli, D. Yoon, A.

Lombardo, A. C. Ferrari, A. Marini, G. Cerullo, and D. Prezzi, ACS Nano 10, 1182-1188 (2016).

${ }^{24}$ P. Steinleitner, P. Merkl, P. Nagler, J. Mornhinweg, C. Schüller, T. Korn, A. Chernikov, and R. Huber, Nano Lett. 17, 1455-1460 (2017). 
${ }^{25}$ U. Keller, Nature, 424, 831-838 (2003).

${ }^{26}$ H. Shi, R. Yan, S. Bertolazzi, J. Brivio, B. Gao, A. Kis, D. Jena, H. G. Xing, and L. Huang, ACS Nano 7, 1072-1080 (2013).

${ }^{27}$ E. M. Mannebach, K. N. Duerloo, L. A. Pellouchoud, M. J. Sher, S. Nah, Y. H. Kuo, Y. Yu, A. F. Marshall, L. Cao, E. J. Reed, and A. M. Lindenberg, ACS Nano 8, 10734-10742 (2014).

${ }^{28}$ T. Borzda, C. Gadermaier, N. Vujicic, P. Topolovsek, M. Borovsak, T. Mertelj, D. Viola, C. Manzoni, E. A. A. Pogna, D. Brida, M. R. Antognazza, F. Scotognella, G. Lanzani, G. Cerullo, and D. Mihailovic, Adv. Funct. Mater. 25, 3351-3358 (2015)

${ }^{29}$ N. Kumar, Q. Cui, F. Ceballos, D. He, Y. Wang, and H. Zhao, Phys. Rev. B 89, 125427 (2014)

${ }^{30}$ D. Sun, Y. Rao, G. A. Reider, G. Chen, Y. You, L. Breźin, A. R. Harutyunyan, and T. F. Heinz, Nano Lett. 14, 5625-5629 (2014).

${ }^{31}$ R. Fleischhaker, N. Krauß, F. Schättiger, and T. Dekorsy, Opt. Express 21, 6764-6776 (2013).

${ }^{32}$ K. M. Dani, J. Lee. R. Sharma, A. D. Mohite, C. M. Galande, P.M. Ajayan, A. M. Dattelbaum, H. Htoon, A. J. Taylor, and R. P. Prasankumar, Phys. Rev. B 86, 125403 (2012).

${ }^{33}$ H. Xia, H. Li, C. Lan, C. Li, X. Zhang, S. Zhang and Y. Liu, Opt. Express 22, 17341-17348 (2014).

${ }^{34}$ M. Seo, H. Yamaguchi, A. D. Mohite, S. Boubanga-Tombet, J. Blancon, S. Najmaei, P. M. Ajayan, J. Lou, A. J. Taylor and R. P. Prasankumar, Sci. Rep. 6 (2016).

${ }^{35}$ B. Liu, Y. Meng, X. Ruan, F. Wang, W. Liu, F. Song, X. Wang, J. Wu, L. He, R. Z. Zhang and Y. Xu, Nanoscale 9, 18546- 18551 (2017). 
(a)

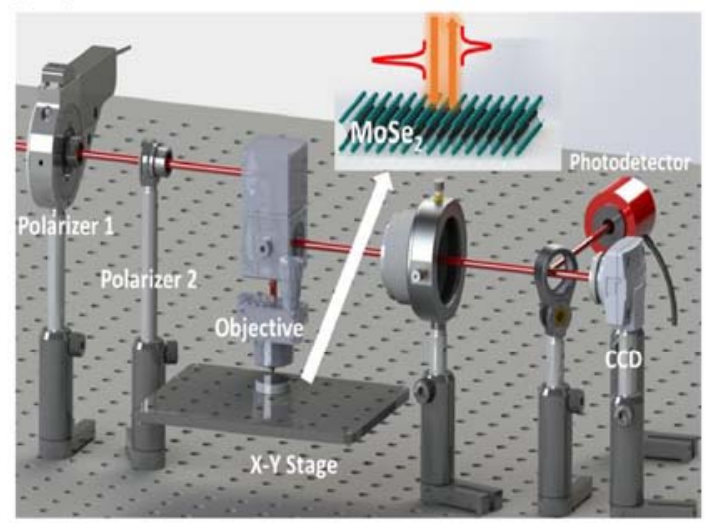

(c)

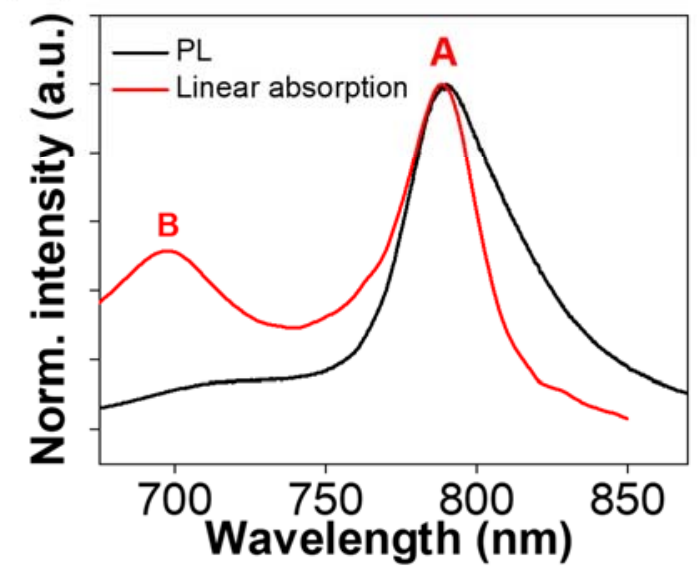

(b)

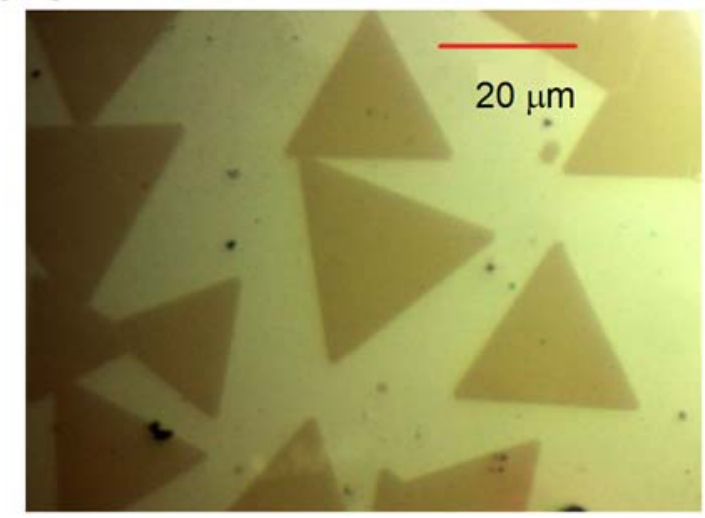

(d)

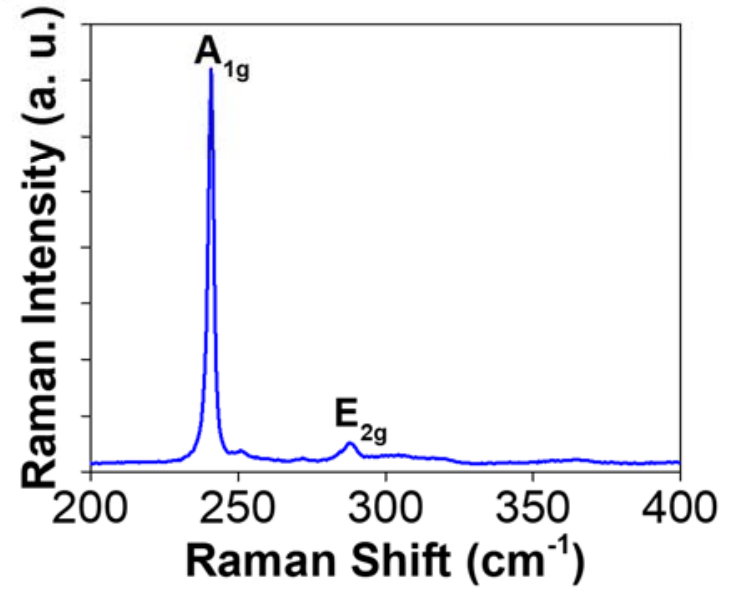

Fig.1. Characterization of monolayer $\mathrm{MoSe}_{2}$ fabricated by CVD. (a) A schematic of the measurement setup. (b) Optical micrograph under white light illumination, scale bar is $20 \mu \mathrm{m}$; (c) Photoluminescence and linear absorption spectrum, and (d) Raman spectrum of the monolayer $\mathrm{MoSe}_{2}$ flake. 
(a)

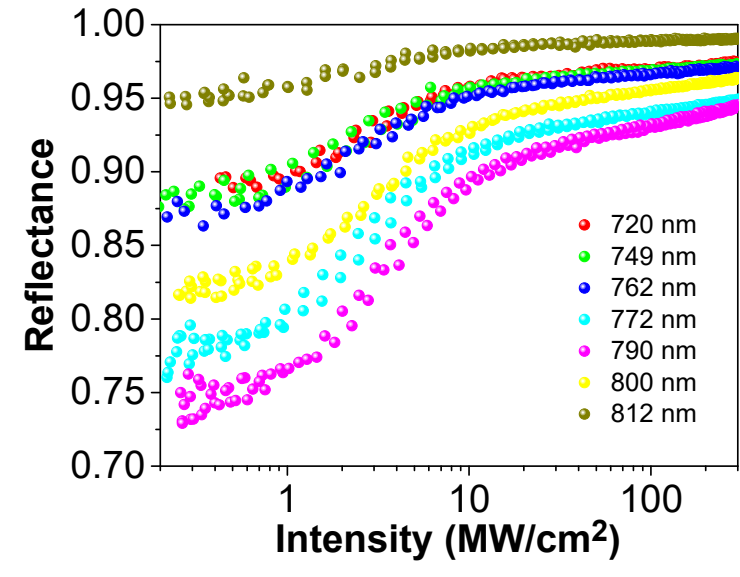

(b)

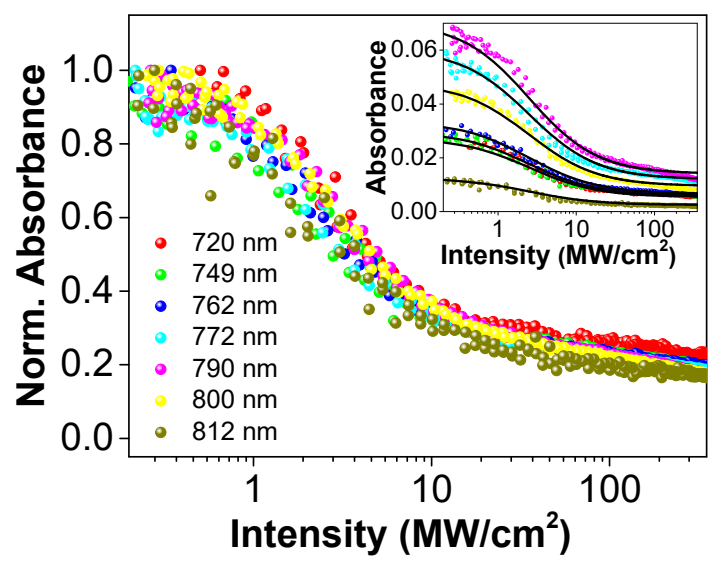

Fig.2.The saturable absorption of monolayer $\mathrm{MoSe}_{2}$. (a) Nonlinear reflectance ( $\mathrm{MoSe}_{2}$ on mirror), and (b) normalized absorption of $\mathrm{MoSe}_{2}$ at various wavelengths; the inset is nonlinear absorbance of $\mathrm{MoSe}_{2}$ with the saturable absorption fittings (black solid lines). 
(a)

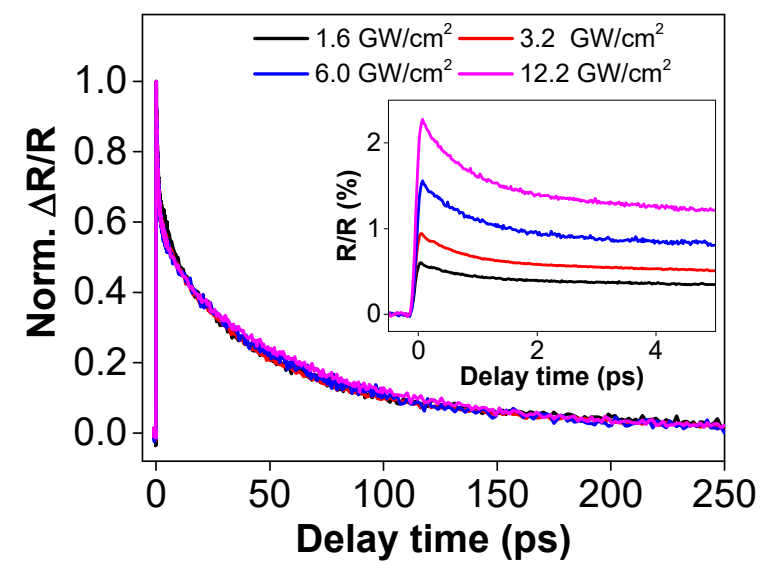

(b)

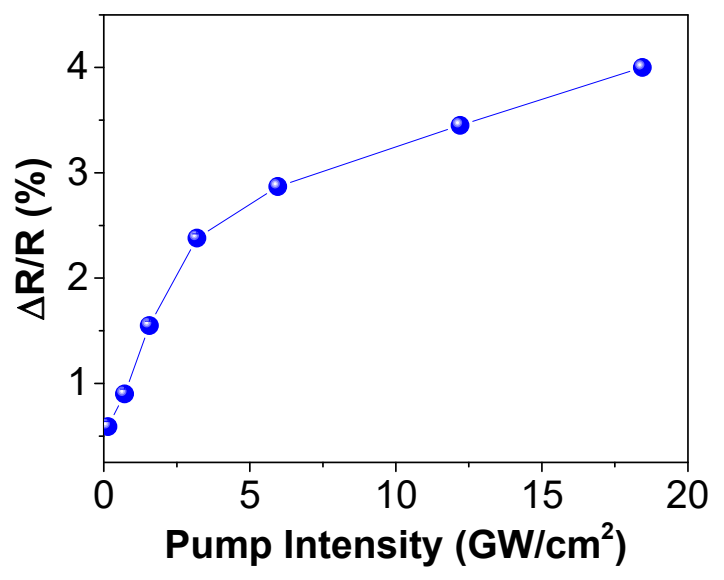

Fig. 3. Recovery time of monolayer $\mathrm{MoSe}_{2}$ by degenerate pump-probe spectroscopy (a) Differential reflection $\Delta \mathrm{R} / \mathrm{R}(\mathrm{t})$ of monolayer $\mathrm{MoSe}_{2}$ measured with pump and probe pulses centered at $760 \mathrm{~nm}$ for different excitation energies. Differential reflectance signal in the first 10 ps in the inset; (b) the peak value of dynamics as a function of pump intensity. 